\title{
OTR9 Effect of zinc on the structural stability of pneumococcal surface antigen $A$ (PsaA)
}

Izabella Buty da Silva Corrêa ${ }^{1}$, Ana Paula Dinis Ano Bom¹, Ana Paula Correa Argondizzo ${ }^{1}$, Ariane Larentis ${ }^{2}$, Marco Alberto Medeiros ${ }^{1}$, José Godinho da Silva Junior ${ }^{1}$

${ }^{1}$ Bio-Manguinhos, Fiocruz, Rio de Janeiro, Brazil

2 ENSP, Fiocruz, Rio de Janeiro, Brazil

Introduction: The Streptococcus pneumoniae is the most common human respiratory pathogen responsible for at least million of deaths worldwide annually. S. pneumoniae has two types of virulence determinants, the heterogenic capsule and the surface proteins. PsaA protein (pneumococcal surface antigen $A$ ) is a virulence factor of $S$. pneumoniae that belongs to the manganese and zinc bacterial transport system. The manganese PsaA binding has been associated with oxidative stress resistance which becomes a pivotal element in the proliferation and virulence of the bacteria. It has been shown that the excess of zinc promote bacteria toxicity since zinc inhibits the acquisition of manganese.

Objective: We have performed a conformational and stability analysis of PsaA recombinant protein in the presence ( $\mathrm{Zn}-\mathrm{PsaA})$ or absence of zinc (free-PsaA), aiming to understand how zinc homeostasis impacts on the host protection and bacteria toxicity.

Methodology: In this study, PsaA was isolated and analyzed by SDSPAGE-12\%. The structural characterization of PsaA $(0.08 \mathrm{mg} / \mathrm{mL})$ was analyzed by fluorescence spectroscopy. We performed a test in the presence of increasing concentrations of zinc $(5-500 \mu \mathrm{M})$ to determine the metal minimum concentration which induces a conformational change. The (Zn-PsaA or free-PsaA) protein stability was observed in the presence of different Urea (1-9 M), Guanidine ( $\mathrm{GdmCl})(1-7 \mathrm{M})$ or salt concentrations $(25-500 \mathrm{mM})$. Moreover, the samples were analyzed in the $\mathrm{pH}$ range (2.6 at 8.0 ) or treated with temperatures from $250 \mathrm{C}$ to $850 \mathrm{C}$.

Results: The experiments in the zinc presence demonstrated that the PsaA conformational changes started at $5 \mu \mathrm{M}$ until $50 \mu \mathrm{M}$ metal concentration. After $50 \mu \mathrm{M}$ the structural modifications were stabilized. The free-PsaA and Zn-PsaA protein presented different unfolding profiles upon chemical denaturation. Conformational change for freePsaA occurs at $1.0 \mathrm{M}$ Urea or $0.5 \mathrm{M}$ of $\mathrm{GdmHCl}$. In contrast, the ZnPsaA unfolding occurs in higher concentrations of Urea $(4 M)$ or $\mathrm{GdmHCl}(1.5 \mathrm{M})$. In the free-PsaA or Zn-PsaA heat denaturation process we verified conformational changes at $43^{\circ} \mathrm{C}$ and $73^{\circ} \mathrm{C}$. It was demonstrated by light scattering that PsaA aggregation is inhibited by $50 \mu \mathrm{M}$ zinc concentrations. To evaluate the PsaA hydrophobic surfasse 
exposure we used bis-ANS probes, but these experiments showed no significant differences between the free or zinc bound protein. Moreover, the $\mathrm{pH}$ experiments showed a decrease of PsaA fluorescence intensity in acid $\mathrm{pH}$ when compared to neutral and basic $\mathrm{pH}$, both in the zinc presence and absence. The free-PsaA, when submitted to salt concentrations, presented a higher center of mass variation when compared to $\mathrm{Zn}-\mathrm{PsaA}$.

Conclusion: Our data shows that free-PsaA has a lower stability if compared to the $\mathrm{Zn}$-PsaA protein when they are subjected to physical or chemical denaturation. This study may contribute to elucidate the mechanism of colonization or inhibition of proliferation dependent of metal in the bacteria.

Keywords: Bioquímica, Caracterização Estrutural 